\title{
Historia de la vegetación en el litoral norte de Girona entre los siglos VIII y XX d.C.: Cambios climáticos y socioeconómicos desde una perspectiva paleoambiental
}

\author{
$\mathrm{H}$ istory of vegetation in the northern coast of Girona: climatic and social- \\ economical changes from a palaeoenvironmental perspective
}

José Antonio López Sáez*; Lourdes López Merino*;

Sebastián Pérez Díaz*; Miguel Ángel Mateo Mínguez ***

\begin{abstract}
RESU MEN
El análisis palinológico de un registro fósil obtenido en la Bahía de Port Lligat (Girona) ha permitido reconstruir la historia de la vegetación litoral del noreste de la Península Ibérica y del Pirineo oriental catalán durante los últimos 1600 años, en directa relación con la antropización de los ecosistemas forestales y los cambios climáticos acontecidos.
\end{abstract}

Palabras clave: Paleopalinología. Paleoambiente. Cambio climático. Holoceno reciente. Girona.

\section{INTRODUCCIÓN}

Los estudios paleopalinológicos cuentan con una larga tradición en el seno de la literatura paleoambiental, permitiendo la reconstrucción de la historia de la vegetación, para intervalos de tiempo relativamente amplios, en directa correlación con la diacronía y dinámica de la antropización de los ecosistemas, la aparición y difusión de especies cultivadas, y el establecimiento de pautas de evolución climática.

En la Península Ibérica, la mayor parte de este tipo de investigaciones se han llevado a

\begin{abstract}
Pollen analysis of a fossil record obtained in the Bay of Port Lligat (Girona) allow us to reconstruct the history of vegetation in the northeast coast of the Iberian Peninsula and the eastern Catalan Pyrenees during the past 1600 years, in direct relation to the man forest management and climate changes that have taken place.
\end{abstract}

Key words: Palaeopalynology. Palaeoenvironment. Climatic change. Late Holocene. Girona.

cabo en yacimientos arqueológicos y en depósitos naturales como lagos y turberas (LÓPEZ SÁEZ et alii, 2003). El inconveniente de los primeros es su adscripción a ventanas cronológicas cerradas que no permiten un estudio detallado sobre la evolución temporal de los bosques; mientras que en el caso de los segundos el interés fundamental de los paleoecólogos se ha centrado en cuestiones de tipo paleofitogeográfico. En segundo término, la mayor parte de este tipo de investigaciones, en yacimientos arqueológicos, han tenido un interés dirigido a contextos prehistóricos y protohistóricos, renunciando en general a periodos posterio-

* Grupo de Investigación Arqueobiología, Instituto de Historia, Centro de Ciencias Humanas y Sociales (CCHS), CSIC, cl Albasanz 26-28, 28037 Madrid.

*** Centre d'Estudis Avançats de Blanes (CEAB), CSIC, Accés a la Cala St. Francesc 14, 17300 Blanes (Girona). 
res como la Edad Media. En paralelo, desde la Arqueología Medieval tampoco se ha solicitado de los paleoecólogos investigaciones de esta índole, por lo que actualmente la bibliografía peninsular adolece de estudios detallados sobre la historia de la vegetación en periodos que se extienden entre el Medievo y la actualidad (IZQUIERDO BENITO, 1994).

El trabajo que se presenta hace referencia al análisis palinológico de un depósito fósil submarino inédito en este tipo de disciplinas, situado en la costa norte de Girona. Su alta resolución cronológica ha permitido una reconstrucción sensible de los principales cambios climáticos acontecidos a lo largo de la Edad Media y hasta la actualidad en el área de estudio, y su relación con diferentes estrategias paleoeconómicas y modos de explotación del medio. Dado el carácter de diáspora que tienen los pólenes y las esporas, el análisis palinológico permite no sólo la reconstrucción de la historia de la vegetación en la zona litoral norte de la provincia de Girona, sino también obtener una visión regional de dicha evolución vegetal de gran parte del Pirineo oriental catalán.

El interés de esta investigación es aportar un nuevo punto de vista al estudio de los paisajes medievales del noreste de la Península Ibérica, a partir del registro fósil, que pueda ser cotejado con otras fuentes documentales de información como son los textos o la propia excavación arqueológica. En segundo lugar, tiene la peculiaridad de ahondar en un marco cronológico poco reconocido en el seno de la bibliografía paleopalinológica peninsular, pues, como se dijo, pocos son los estudios detallados al respecto.

\section{METODOLOGÍA}

El análisis palinológico presentado en este trabajo procede de un depósito fósil submarino muy peculiar, la denominada 'mata' de la fanerógama marina Posidonia oceanica en la Bahía de Port Lligat en Girona (Fig. I). Un sondeo de $120 \mathrm{~cm}$ de potencia estratigráfica ha permitido obtener un registro sedimentario de la historia de la vegetación del área para los últimos 1600 años con un grado de resolución aproximado de 3-4 cm excepto para la parte basal. Detalles concernientes a la metodología de su muestreo pueden encontrarse en MATEO et alii (1997). Acerca de la formación de este tipo de depósitos, y las caracterísitcas de su proceso de acumulación, existen ya algunas publicaciones previas de los miembros de este trabajo que pueden ser consultadas (ROMERO et alii, 1994; MATEO et alii, 1997, 2002).

Cinco dataciones carbono- 14 han sido realizadas, en distintos tramos del registro, en el National Ocean Sciences AM S Facility (W oods Hole Oceanographic Institution). A partir de ellas se ha elaborado un modelo cronológico interpolando los ritmos de acumulación entre cada datación (Figs. 2-3). Las fechas BP fueron convertidas en sus respectivas fechas calibradas en años d.C. mediante el programa Calib v.5.0.2. En la Fig. 3 la cronología se expresa tanto en términos de su edad carbono- 14 convencional (fechas no calibradas BP -before present- tomando el año 1950 d.C. como el presente, con su respectiva desviación estándar), como en fechas estimadas calibradas d.C. obtenidas mediante una interpolación lineal entre las fechas BP disponibles gracias al modelo cronológico (Fig. 2). Más detalles sobre la cronología de este depósito, el modelo edad-profundidad considerado, y la calibración de las fechas (incluyendo el efecto reservorio estimado en este trabajo respecto al mar Mediterráneo), se encuentran en MATEO et alii $(1997,2002)$.

Todas las muestras polínicas fueron tratadas en el Laboratorio de Arqueobiología del $\mathrm{CCH}$ (CSIC, Madrid), siguiendo la metodología química tradicional de esta disciplina (FAEGRI, IVERSEN, 1989). Los pólenes, esporas y otros microfósiles no polínicos fueron identificados utilizando claves morfológicas, hasta una suma base polínica de casi 500 granos, excluyendo de esta cantidad los hidro-higrófitos (por su sobrerrepresentación en estos medios) así como los palinomorfos no polínicos. Una vez estudiadas todas las muestras se confeccionó el diagrama polínico correspondiente (Fig. 3), para cuya elaboración nos servimos del programa Tilia y Tilia-Graph v. 2.0.b.5. Un análisis estadístico 
mediante el método aglomerativo propuesto por el programa Coniss (GRIMM, 1992) ha permitido definir en él una serie de zona polínicas (I a 8) que señalan los principales cambios acontecidos en la vegetación a lo largo del tiempo. La lectura diacrónica de dichas zonas polínicas permite una interpretación paleoecológica del registro más sencilla y detallada.

\section{RECONST RUCCIÓN DEL PALEOPAISAJE}

\section{Siglos VIII-X d.C. (zona 1)}

La zona polínica I ofrece datos sobre la historia de la vegetación entre los siglos VIII y $X$ d.C. (Fig. 3). Aunque el modelo cronológico (Fig. 2) sitúe el inicio de esta zona hacia el 840 d.C., la calibración de la fecha basal de depósito fósil de $1600 \pm 45$ BP (724-954 d.C.) nos obligaría a considerar que la zona polínica I pudo en cualquier caso iniciarse en la segunda mitad del siglo VIII d.C.

Entre ca. 750- 1000 d.C. la vegetación arbórea predominante en el área de estudio correspondió a formaciones caducifolias de Quercus pyrenaica tipo, acompañadas de Q. suber, es decir a robledales y alcornocales. El porcentaje conjunto de ambos palinomorfos (ca.20\%) permitiría suponer la existencia de un robledal con alcornoque relativamente abierto, en el seno del cual ciertos elementos mesófilos como el abedul (Betula) o el castaño (Castanea) podrían tener cierta importancia. El carácter heliófilo del abedul habría, seguramente, facilitado su relativa abundancia en estos momentos en los que el bosque climácico regional, el robledalalcornocal, no tenía demasiada densidad.

En el caso del castaño, a pesar de apenas presentar un porcentaje inferior al 1\%, lo más probable es que su presencia esté referida al cultivo regional de dicha especie, toda vez que Castanea sativa fue cultivada en la región al menos desde el 2700 BP (BURJACHS, SCHULTE, 2003; BURJACHS, 2004; CONEDERA et alii, 2004; BURJACHS et alii, 2005).
Aún encontrándonos en una zona costera, el escaso bagaje porcentual de Pinus halepensis tipo $(<5 \%)$ sería indicativo del poco desarrollo de pinares costeros de pino carrasco, termomediterráneos en el área de estudio. En cambio, el taxón arbóreo mayoritario, en esta biozona del diagrama polínico es Pinus sylvestris tipo con porcentajes que desde el $20 \%$ en la base se van elevando progresivamente hasta superar el 30\% justo antes del año I000 d.C. Estos datos estarían indicando, posiblemente, un importante desarrollo de pinares (Pinus sylvestris y $P$. uncinata) en las estribaciones montañosas pirenaicas centro-orientales (Sierra del Cadí, Sierra del Catllar, etc.), ya que dado el carácter anemófilo de este palinomorfo es normal que sus porcentajes sean significativos sin ser excesivamente elevados.

La vegetación arbustiva, en este marco cronológico, tendría una notable importancia, con un desarrollo significativo de brezales de Erica arborea tipo y jarales de Cistus ladanifer, así como de una maquía xerotermófila compuesta principalmente de mirto (Myrtus), Phillyrea y acebuche (Olea europaea). Brezales y jarales constituirían el matorral propio de las etapas degradativas del bosque local, el robledalalcornocal, lo cual dada su relativa importancia permitiría explicar el estado de bosque abierto antes comentado para dicha formación forestal, en cuyos claros prosperaría un amplio dosel arbustivo. La maquía xerotermófila ocuparía probablemente las estaciones más xéricas y soleadas, sobre litosuelos o en zonas de escaso desarrollo edáfico donde los elementos arbóreos difícilmente prosperarían.

Los datos hasta ahora expuestos se ajustan perfectamente a la vegetación potencial del área (RIVAS MARTÍNEZ, 1987; FRANQUESA, 1995), el alcornocal mesomediterráneo acidófilo y subhúmedo de Quercus suber, en el cual ciertos elementos arbustivos como Erica arborea o Phillyrea juegan un papel muy importante en sus etapas de sustitución. La antropización de la región, en este marco cronológico, habría favorecido la extensión de los brezales en detrimento de las formaciones forestales de quercíneas. De hecho, la situación costera del registro habría permi- 
tido una mayor preponderancia de elementos termófilos, como el mirto o el acebuche, que caracterizan, precisamente, la faciación termófila del alcornocal. La escasa importancia cobrada por Quercus ilex tipo en estos momentos se explica teniendo en cuenta que este tipo de bosques (encinar y/o alsinar) apenas cuenta con representaciones residuales en el área de estudio y en general en la zona septentrional de Cataluña, donde el dominio fisionómico del paisaje corresponde al alcornocal.

Entre la flora herbácea dominan los elementos xerófilos (Artemisia, Chenopodiaceae/ Amaranthaceae) y aquéllos mediados por la antropización del territorio (Aster tipo, Cardueae, Cichorioideae, Rumex acetosa tipo, etc.); aunque es importante señalar algunos que tienen relación con actividades pastoriles o pastizales antropozoógenos (Plantago lanceolata tipo, Urtica dioica tipo, etc.) (BEHRE, 1981; GALOP, 1998). Probablemente estos palinomorfos antrópicos serían el mejor indicativo de la antropización consiguiente de las formaciones forestales y su estado de bosques abiertos antes referido. En concordancia con lo anterior, la presencia de Sordaria sp en estas muestras corroboraría la presión pastoral citada, toda vez que se trata de ascosporas fúngicas de especies en su mayoría de ecología coprófila (LÓPEZ SÁEZ et alii, 1998, 2000; VAN GEEL, 200I).

\section{Siglo XI d.C. y primera mitad del siglo XII d.C. (zona 2)}

Entre los años 1000 d.C. y ca. II 50 d.C., correspondiendo a la zona polínica 2 (Fig. 3), se produce una drástica deforestación del pinar altimontano que puebla los Pirineos orientales catalanes; la cual viene acompañada por la finalización del cultivo del castaño y cierta progresión de elementos heliófilos como el abedul (7\%). Este proceso también parece afectar al bosque de quercíneas de la costa y valles gerundenses, pues tanto Quercus pyrenaica tipo como Q. suber disminuyen ligeramente sus porcentajes.

En paralelo, este ingente proceso deforestador provoca una gran extensión de los brezales (Erica arborea tipo) y enebrales/ sabinares (Juniperus tipo); y en menor medida de pastizales de origen antrópico (Aster tipo, Cichorioideae, etc.). De alguna manera parece ser que estuvo centralizado en los pinares pirenaicos, con mucha menor importancia en las zonas litorales y prelitorales, aunque éstas (alcornocales y robledales) también parecen sufrir su influjo.

Es curioso señalar que algunos palinomorfos herbáceos de origen antropozoógeno, caso de Chenopodiaceae/Amaranthaceae, Plantago lanceolata tipo o Urtica dioica tipo, e incluso Sordaria sp entre los microfósiles no polínicos, desaparezcan a lo largo de este intervalo (siglos XI-XII d.C.) de la zona polínica 2; por lo que este proceso también pareció implicar una desaceleración en el trasiego local y regional de cabañas pastoriles.

En general, los hechos antes expuestos parecen estar refiriendo, sobre todo, un fuerte impacto antrópico deforestador sobre los pinares pirenaicos del NE de la Península Ibérica, que cronológicamente parece coincidir con un aumento notable del poblamiento medieval e importantes cambios sociales en el Pirineo catalán (BONNASSIE, 1988, 1990; RIU, 2003). En este sentido, el registro fósil de Port Lligat estaría reflejando perfectamente ese aumento poblacional de la montaña pirenaica y la deforestación provocada sobre el pinar altimontano a causa de ello. Por lo tanto, el análisis palinológico demuestra aquí su versatilidad para reconstruir la historia de la vegetación no sólo en un ámbito meramente local (costero o litoral) sino también en una escala regional que implicaría el Pirineo oriental.

A la vez que esa manifiesta antropización y deforestación se producía en el Pirineo, en este mismo intervalo cronológico aconteció un cambio climático hacia condiciones de mayor aridez, lo cual podría haber incidido también en la evolución de los bosques de la zona de estudio. Este cambio climático abrupto podría ser el responsable de la disminución de la presión pastoral que se manifiesta en el entorno inmediato de la Bahía de Port Lligat. 
En conclusión, es probable que estos hechos citados tengan relación con el inicio del denominado Periodo Cálido Medieval, que se extendió entre ca. 900-I 360 d.C. (RIERA et alii, 2004, 2006; BEAULIEU et alii, 2005); o más probablemente con un mínimo de actividad solar en el seno de dicho periodo, el denominado Mínimo de Oort (I0I0-I050 d.C.), en el cual se produjeron condiciones frías y áridas (DESPRAT et alii, 2003) que podrían explicar, por ejemplo, la reducción porcentual de Olea europaea en este tramo de la secuencia.

Un evento semejante al descrito ha sido documentado en el registro paleoambiental del lago Estanya (RIERA et alii, 2004, 2006) entre los años 820 d.C. y 1075 d.C., donde la continua recurrencia de incendios antrópicos habría conducido a una enorme deforestación semejante a la citada en Port Lligat, mediada sobre todo por un mayor impacto de las actividades ganaderas, así como con una disminución del nivel lacustre a causa de condiciones climáticas más áridas. Este evento documentado en Port Lligat ha sido igualmente ilustrado en otros registros polínicos de la provincia de Girona, demostrándose su correlación con talas de los bosques de la región pirenaica del NE de la Península Ibérica a partir del siglo XI d.C. (MALLARACH et alii, 1985; PĖLACHS, 2004).

\section{Segunda mitad del siglo XII d.C. a siglo XIV d.C. (zona 3)}

A partir de la segunda mitad del siglo XII d.c. (ca. II50 d.C.), el pinar se recupera lo mismo que las quercíneas caducifolias, se vuelve a cultivar el castaño, y aparece el haya (Fagus) por primera vez en el diagrama polínico, todo ello en la zona polínica 3 (Fig. 3). El bajo porcentaje de haya $(<1 \%)$ permite suponer su carácter regional a partir de poblaciones que podrían estar ubicadas en el Pirineo oriental o en algunas sierras litorales catalanas.

Entre ca. II 50 d.C. y 1380 d.C. (zona 3) las formaciones forestales del área de estudio siguen siendo las mismas que en periodos anteriores, es decir bosques de quercíneas (alcornoques y robles). Si bien el robledal parece ir perdiendo progresivamente su importancia, ocurre algo parecido con el alcornoque (Quercus suber) que se mantiene relativamente constante todo el tiempo con tendencia a la regresión. La suma porcentual de ambos morfotipos polínicos sigue estando en torno al $20 \%$, lo que volvería a señalar las características de bosque local relativamente abierto, en el seno del cual prosperarían ciertos elementos mesófilos como el abedul (Betula), mientras que se cultiva de nuevo el castaño (Castanea sativa).

Las formaciones arbustivas siguen estando dominadas por brezos (Erica arborea tipo), jaras (Cistus ladanifer) y ahora también por enebros/ sabinas (Juniperus tipo), si bien los primeros tienen mucha menor importancia que en los siglos anteriores, mientras que las segundas mantienen porcentajes constantes relativamente más altos. En cualquier caso, su persistencia seguiría demostrando la importancia de estos elementos arbustivos en las etapas de sustitución del bosque de quercíneas. La maquía xerotermófila se reduce ahora a Phillyrea y probablemente a alguna especie del género Juniperus. Aunque Olea europaea sigue estando presente, cabe la posibilidad de que este palinomorfo esté haciendo referencia tanto al acebuche como elemento de dicha maquía como al cultivo local del olivo. Esto último parece lo más factible si se considera una perspectiva regional de la cuestión (PANTALEÓN-CANO et alii, 1994; BURJACHS, 2004), y si se tiene en cuenta que en este marco cronológico sus porcentajes se incrementan notablemente $(>5 \%)$.

En resumen, el alcornocal mesomediterráneo acidófilo y subhúmedo seguiría siendo el bosque que caracterizaría las formaciones forestales locales, igualmente antropizado que en los siglos anteriores. La persistencia de la maquía xerotermófila seguiría caracterizando la faciación más térmica del alcornocal, aunque la desaparición del mirto (M yrtus) permitiría suponer cierta incidencia antrópica sobre ésta.

La flora herbácea está dominada fundamentalmente por elementos de carácter nitrófilo y antrópico (Aster tipo, Cardueae, Cichorioideae) o antropozoógeno (Chenopodiaceae/ Amaran- 
thaceae, Plantago lanceolata tipo, Urtica dioica tipo) (BEHRE, 1981; GALOP, 1998), con porcentajes superiores a los siglos anteriores, todo lo cual señalaría que la antropización de estos territorios entre la segunda mitad del siglo XII d.C.y el siglo XIV d.C. fue mucho más importante que en los precedentes; incluyendo una carga pastoral reseñable, igualmente demostrada por la importancia que cobran ahora ciertos hongos de ecología coprófila asignables al género Sordaria (LÓPEZ SÁEZ et alii, 1998, 2000;VAN GEEL, 200 I).

La importancia que cobran dos microfósiles no polínicos como Pseudoschizaea circula y Glomus cf. fasciculatum, relacionados con el desarrollo de procesos erosivos, permitiría estimar su presencia en directa relación con la erosión originada por la actividad del oleaje sobre el ambiente costero local (ANDERSON et alii, 1984) como con los procesos de esta índole que localmente produciría la antropización del bosque de alcornoques y robles (LÓPEZ SÁEZ et alii, 2000).

Entre ca. 1280 d.C. y $\mid 340$ d.C., casi al final de la zona polínica 3, se vislumbra una disminución porcentual o desaparición de la mayor parte de palinomorfos arbóreos (abedul, castaño, Pinus sylvestris tipo) y arbustivos (Cistus ladanifer, Erica arborea tipo), y un ligero aumento de herbáceas antrópicas (Aster tipo, Cichorioideae). Este pequeño intervalo muestra, en paralelo, el cese de toda actividad pastoril localmente, ya que desaparecen Plantago lanceolata tipo, Urtica dioica tipo y Sordaria sp, palinomorfos indicativos de tal actividad (GALOP, 1998; LÓPEZ SÁEZ et alii, 2000). En conjunto, estos datos supondrían una ralentización notable de la presión antrópica sobre el paisaje, particularmente de la pastoral, seguramente mediada por condiciones más áridas y frías; las cuales podríamos relacionar con otro mínimo de actividad solar en el seno de la Pequeña Edad del Hielo, el denominado Mínimo de Wolf (DESPRAT et alii, 2003).

\section{Primera mitad del siglo XV d.C. (zona 4)}

La dinámica general de reforestación experimentada en los dos siglos y medio anteriores se ve drásticamente interrumpida a finales del siglo XIV d.C. (ca. I 380 d.C.) y durante toda la primera mitad del siglo XV d.C. (hasta el I 450 d.C.), en la zona polínica 4 (Fig. 3).

En este intervalo cronológico, de apenas 50-70 años, se produce una disminución porcentual muy importante de la mayor parte de los elementos arbóreos principales, caso de los pinos pirenaicos (Pinus sylvestris tipo), el abedul (Betula), los robles (Quercus pyrenaica tipo), e incluso la desaparición temporal de otros como el castaño. Tal reducción parece también afectar a la cobertura arbustiva, pues tanto las jaras (Cistus ladanifer), los enebros/ sabinas (Juniperus tipo), como los brezos (Erica arborea tipo), reducen sus valores porcentuales, especialmente en el caso de estos últimos.

El final del siglo XIV d.C. y la primera mitad del siglo $X V$ d.C. suponen pues un momento álgido de la antropización del paisaje, toda vez que los porcentajes de algunos elementos nitrófilos y antrópicos, como Cichorioideae, aumentan espectacularmente (>40\%) sus porcentajes.

Los modelos de explotación del medio en este marco cronológico parecen responder, principalmente, a una explotación más importante de éste por parte del ganado, pues ahora las evidencias de una mayor presión pastoral se incrementan: máximos para ciertos palinomorfos antropozoógenos como Plantago lanceolata tipo o Urtica dioica tipo (GALOP, 1998), o para algunos microfósiles no polínicos indicativos de dichas actividades caso de Sordaria sp o Riccia cf. sorocarpa (LÓPEZ SÁEZ et alii, 1998, 2000; CARRIÓN, NAVARRO, 2002).

La documentación de Chaetomium sp, exclusivamente en este intervalo a lo largo de toda la secuencia polínica, permitiría relacionar este microfósil no polínico -correspondiente a ascosporas fúngicas de especies carbonícolas (LÓPEZ SÁEZ et alii, 1998)- con el desarrollo de procesos de incendio que hubieran producido la deforestación comentada.

Este nuevo evento, que cronológicamente podríamos situarlo a ca. I 380- I 450 d.C., supone, en resumen, una pérdida notable de cober- 
tura forestal (árboles y arbustos), que parece venir mediada por la incidencia de procesos de incendio de origen antrópico, por un aumento de la carga pastoral, y, en general, por un incremento muy importante de la antropización del paisaje de la zona de estudio.

En otros registros polínicos del NE de la Península Ibérica (RIERA et alii, 2004, 2006), entre I 360 d.C. y I580 d.C. se asiste a un aumento de los niveles lacustres, coincidente con otro episodio frío y de baja actividad solar de la Pequeña Edad del Hielo (|400-| 850 d.C.), el denominado Mínimo de Spörer (| 420-I530 d.C.) (DESPRAT et alii, 2003; BEAULIEU et alii, 2005). Es más que probable que este nuevo detrimento climático también fuera responsable del declive de las formaciones forestales, arbóreas y arbustivas, solapándose su efecto al de una dinámica antrópica creciente.

\section{Segunda mitad del siglo XV d.C a primera mitad del siglo $X V I$ d.C. (zona 5)}

Durante la segunda mitad del siglo XV d.C. y las primeras décadas del siglo XVI d.C. (I450-I530 d.C.), los espectros polínicos de la zona 5 (Fig. 3) demuestran un auge muy importante de los pinares pirenaicos, pues Pinus sylvestris tipo alcanza su porcentaje más alto de todo el registro fósil (55\%). Se estaría por tanto produciendo la reforestación del Pirineo oriental de Cataluña, seguramente tras el evento climático árido ocurrido en la primera mitad del siglo XV d.C. y tras cesar la fuerte antropización de estos entornos montañosos. De hecho, es en estos momentos, a inicios del siglo XVI d.C., cuando se detecta por primera vez en el diagrama polínico la presencia de abeto (Abies), avellano (Corylus) y la reaparición del haya (Fagus).

En la zona litoral, los robledales van poco a poco cobrando menor importancia, mientras que el alcornoque llega incluso a desaparecer al iniciarse el siglo XVI d.C.

El declive de los bosques litorales se circunscribe en un periodo en el cual la antropi- zación de los ambientes costeros experimenta un nuevo desarrollo, volviéndose a cultivar el castaño y posiblemente el olivo. De hecho, el impacto antrópico tuvo que ser lo suficientemente alto para afectar también a las etapas degradativas del bosque, pues tanto brezos como jaras se ven sensiblemente afectados, y vuelven a documentarse importantes eventos erosivos asociados a un máximo porcentual de Pseudoschizaea circula.

Al tratarse de un periodo relativamente corto de tiempo, de unos 80 años, las causas de tal declive de las formaciones caducifolias de quercíneas no quedan demasiado claras, aunque tuvieron que ser la antropización (presencia de nitrófilas como Aster tipo, Cardueae, Cichorioideae, Rumex acetosa tipo) y la recurrencia de la presión pastoral (importancia de ciertos antropozoógenos y hongos coprófilos como Plantago lanceolata tipo, Urtica dioica tipo y Sordaria sp) las principales causas. En todo caso, parece quedar claro que en este marco cronológico el impacto humano sobre el paisaje se centra, a diferencia de siglos anteriores, en las zonas litorales, no viéndose afectado el Pirineo oriental.

\section{Segunda mitad del siglo XVI d.C.- Siglo XVII d.C. (zona 6)}

A partir de la segunda mitad del siglo XVI d.C. el paisaje comienza a sufrir cambios muy importantes en la fisionomía de los bosques, no sólo en el entorno próximo de Port Lligat sino también en el ámbito de los Pirineos orientales.

En la zona polínica 6 (Fig. 3) comienzan a aparecer en el diagrama polínico toda una serie de elementos que indican claramente el desarrollo de actividades de arboricultura y agricultura. En particular, el castaño (Castanea) parece cultivarse ahora con mayor intensidad que antes, y en paralelo empieza el cultivo del nogal (Juglans), la vid (Vitis), el cáñamo (Cannabis/H umulus) y los cereales (Cerealia); sin descartar que una mayor profusión de Olea europaea pueda ser correlacionada también con una mayor intensidad del cultivo del olivo. 
En el intervalo ca. 1530-1700 d.C., como resultado de los cultivos antes comentados, el bosque local de alcornoques y robles se ve seriamente afectado, hasta tal punto que Quercus suber desaparece completamente del registro y Q . pyrenaica tipo reduce sus porcentajes a apenas un 5\%. En paralelo a ello, la alsina o encina (Quercus ilex tipo) cobra ahora mayor importancia, sin que ello deba interpretarse únicamente como resultado del desarrollo de sus bosques sino puede que también de una mayor representativad porcentual a causa de la desaparición progresiva de las otras quercíneas (RIERA, ESTEBAN, 1994).

Tal proceso antrópico conduciría a un mayor desarrollo de formaciones arbustivas de etapas de sustitución del bosque como las que aparecen en la zona 6, dominadas por ciertos elementos xerotermófilos como Olea, Phillyrea y lentisco (Pistacia lentiscus); brezos y jaras.

Se trataría pues de un periodo con un impacto antrópico sobre el paisaje relativamente elevado, como demuestra la preponderancia de ciertos elementos nitrófilos y malas hierbas de cultivos como Aster tipo, Cardueae, Cichorioideae, Convolvulus arvensis tipo y Rumex acetosa tipo entre otros (BEHRE, 1981). Aún es perceptible cierta presión pastoral, toda vez que elementos antropozoógenos son documentados, como Chenopodiaceae/ Amaranthaceae, Plantago lanceolata tipo o Urtica dioica tipo (GALOP, 1998), o microfósiles no polínicos coprófilos como Sordaria sp (LÓPEZ SÁEZ et alii, 1998).

En las zonas pirenaicas orientales la situación es radicalmente distinta, pues la mayor parte de las especies experimentan un auge notable, caso del abeto (Abies) o el haya (Fagus), e incluso de otros elementos mesófilos como el abedul (Betula) o el avellano (Corylus). Indudablemente estos datos están indicando una escasa alteración antrópica de los bosques pirenaicos y en cambio un mantenimiento sostenible de éstos. De hecho, el elemento mayoritario, Pinus sylvestris tipo, mantiene porcentajes relativamente altos (ca. 30\%).
La preponderancia de los cultivos origina procesos erosivos del nivel superficial del suelo, paleoambientalmente señalados por la documentación de Pseudoschizaea circula y Glomus cf. fasciculatum (LÓPEZ SÁEZ et alii, 2000; BURJACHS, SCHULTE, 2003). Estos procesos también pueden estar vinculados a condiciones climáticas más áridas y posiblemente más térmicas, como demuestra la importancia cobrada por elementos xerófilos y termófilos como Artemisia, Phillyrea o Pistacia lentiscus.

\section{Siglo XVIII d.C. a primera mitad del siglo $X I X$ d.C. (zona 7)}

En la zona polínica 7 (Fig. 3), en el intervalo 1700-1840 d.C., los bosques pirenaicos se ven alterados de manera significativa, pues el abeto desaparece, el haya se reduce, y el pinar altimontano sufre una degradación importante. En paralelo, aparece por primera vez un elemento submediterráneo como el boj (Buxus), e incluso Ligustrum, como mejores exponentes de las formaciones arbustivas degradativas de dichos bosques.

Hacia la costa y el fondo de valle se sigue cultivando el castaño y el nogal, la vid y el cáñamo, los cereales y el olivo, éstos últimos con mayor profusión, pues incluso en el caso de los cereales su porcentaje es superior al $3 \%$, suficiente para admitir la existencia local de cultivos (LÓPEZ SÁEZ et alii, 2003). Esta mayor incidencia de los cultivos llega a alterar incluso los bosques de Quercus ilex tipo hasta hacerlos desaparecer del registro polínico; mientras que los elementos antrópicos nitrófilos y antropozoógenos de los siglos precedentes siguen siendo muy abundantes, en particular Cichorioideae, Plantago lanceolata tipo y Sordaria sp.

Como en la zona 6, en ésta se siguen produciendo procesos erosivos relacionados con la recurrencia de los cultivos y el impacto antrópico, por lo que de nuevo se documentan dos microfósiles no polínicos demostrativos de tales hechos, caso de Pseudoschizaea circula y Glomus cf. fasciculatum (PANTALÉON-CANO et alii, 1994; LÓPEZ SÁEZ et alii, 2000; BURJACHS, SCHULTE, 2003). 
Hay que señalar, finalmente, que en el tránsito entre los siglos XVII d.C.y XVIII d.C., aproximadamente entre ca. 1645 - 17 I 5 d.C., el cultivo del castaño se reduce sensiblemente y el de la vid también parece afectado, mientras que cesa completamente toda actividad de cerealicultura; desaparecen o se reducen los porcentajes de muchos elementos arbóreos (abedul, avellano, haya, pino, roble), y se incrementan los de aquéllos indicativos de condiciones más frías y/o áridas (abeto, encina) incluyendo ciertos elementos arbustivos (Phillyrea). Entre la flora herbácea los indicadores de antropización son poco evidentes. En síntesis, estos datos apuntarían hacia un periodo de tiempo relativamente corto, de condiciones climáticas frías y áridas, durante el cual la antropización del paisaje disminuye bruscamente, lo cual implica una ralentización de los cultivos e incluso el abandono de alguno de ellos. Cronológicamente, este intervalo coincide con el denominado Mínimo de Maünder, un periodo especialmente frío y árido de la Pequeña Edad del Hielo (DESPRAT et alii, 2003), quizá el más evidente y drástico de toda ella.

\section{Segunda mitad del siglo XIX d.C.- Siglo XX d.C. (zona 8)}

El tramo final del diagrama polínico (zona 8), correspondiente al intervalo cronológico situado entre 1840 d.C. y el presente (Fig. 3), demuestra una recuperación paulatina del bosque de robles y alcornoques, así como del de encinas/alsinas, al cesar por completo el cultivo de la vid, los cereales, el cáñamo y el nogal, y reducirse muy sensiblemente el del castaño y en menor medida el del olivo.

Los indicadores polínicos de antropización son ahora mucho menos evidentes, desapareciendo la mayor parte de ellos o reduciendo notablemente sus porcentajes como ocurre con Aster tipo, Cichorioideae, etc. No obstante, la presión pastoral sigue existiendo, con más intensidad incluso que en momentos previos, pues se documentan de nuevo ciertos elementos antropozoógenos o coprófilos como Plantago lanceolata tipo, Urtica dioica tipo y Riccia cf. sorocarpa (GALOP, I998; CARRIÓN, NAVARRO, 2002).
En la zona pirenaica los porcentajes de haya se reducen sensiblemente aun cuando esta especie sigue siendo documentada; mientras que el abeto está completamente ausente. El pinar de Pinus sylvestris tipo, en cambio, muestra cierto auge, acercándose a valores porcentuales del $30 \%$.

\section{CONCLUSIONES}

El noreste de la Península Ibérica es un territorio relativamente bien conocido en referencia a la historia de su vegetación durante el Holoceno. La mayor parte de secuencias paleopolínicas disponibles proceden de registros lacustres O yacimientos arqueológicos (BURJACHS et alii, 1990; RIERA, ESTEBAN, 1994). Nuestro estudio es el primero que se emprende en este amplio territorio con una resolución cronológica muy detallada respecto a la Edad Media, siendo perfectamente comparable con otros registros polínicos regionales (MALLARACH et alii, 1985; PÉREZ OBIOL et alii, 1991; PANTALÉON-CANO et alii, 1994; RIERA, ESTEBAN, 1994; BURJACHS et alii, 2005).

Este trabajo se enmarca, precisamente, en el seno de las reconstrucciones paleoambientales llevadas a cabo en Cataluña, complementando e integrando registros polínicos e históricos que permiten corroborar los hallazgos arqueológicos de periodos medievales (MENCHON I BES, 1998; MAURI, 2006); y que hasta el momento únicamente habían sido desarrollados en el Penedés, Garraf y el Llano en la provincia de Barcelona o en el Ampurdán de la de Girona (RIERA, PALET, 1993; ESTEBAN, RIERA, 1994; PALET, RIERA, 1994, 1997; PANTALÉON-CANO et alii, 1994; RIERA, 1999; BURJACHS, SCHULTE, 2003; BURJACHS, 2004; BURJACHS et alii, 2005).

La secuencia polínica de Port Lligat, en un área bajo la influencia del clima mediterráneo, sugiere la presencia de un paisaje relativamente forestado durante los últimos 1600 años, caracterizado por la dominancia de las formaciones de quercíneas (robledales, alcornocales, encinares/alsinares) en los valles y zonas más cercanas a la costa, así como por pinares, abetares y hayedos en las estribaciones montañosas pirenaicas. 
La importancia de las formaciones arbustivas de brezos y jaras, en toda la secuencia, ofrece pruebas sustanciales de la antropización de estos territorios en dicho recorrido cronológico, lo cual se ve corroborado por la abundancia de elementos herbáceos de carácter nitrófilo y antropozoógeno. La riqueza por igual de elementos arbustivos característicos de la maquía xerotermófila (M yrtus, Pistacia lentiscus, Phillyrea, Olea europaea, etc.) demostraría la correspondencia de los bosques de la zona de estudio con la faciación más termófila del alcornocal.

En general, a lo largo de los últimos 1600 años el entorno inmediato de la Bahía de Port Lligat estuvo sometido a un proceso de antropización relativamente importante, donde el impacto de la ganadería tuvo un papel relevante. No obstante, es importante señalar el carácter local o extra-local que en ocasiones tienen los registros polínicos, especialmente en la diagnosis de indicios de antropización, por lo que los datos no siempre son extrapolables entre distintas zonas geográficas.

Por ejemplo, durante el siglo XI d.C. y la primera mitad del siglo XII d.C., el registro polínico de Port Lligat demuestra una desaceleración de la presión pastoral en la zona litoral y en cambio una fuerte antropización de los ambientes pirenaicos. Si bien la primera podemos relacionarla con un cambio climático abrupto en el seno del Periodo Cálido Medieval, la segunda parece venir mediada por un fuerte proceso migratorio poblacional hacia el Pirineo. En el Llano de Barcelona, en este mismo intervalo cronológico, se produce una progresión de las formaciones arbóreas (RIERA, PALET, 1993; RIERA, 1999), posiblemente porque el paisaje se encontraba mucho menos antropizado, lo que lleva a preguntarnos si hubo algún cambio en la tipología o intensidad de las actividades agrícolas y ganaderas y si la recolonización forestal se produce o no sobre antiguos campos cultivados (MAURI, 2006). Precisamente en el Llano de Barcelona son la alsina y el pino carrasco los elementos arbóreos que parecen experimentar mayor desarrollo, lo cual estaría de acuerdo con el registro de Port Lligat en el sentido de admitir condiciones climáticas más áridas.
En la costa gerundense de Port Lligat, el bosque de alcornoques y robles está ampliamente influido por las actividades del ser humano durante los siglos VIII a XII d.C., siendo la ganadería el principal factor de deforestación de la masa forestal ya que apenas parece cultivarse el castaño y posiblemente el olivo. En las comarcas barcelonesas del Penedés y el Garraf, en cambio, entre los siglos VII, VIII y XII d.C., se asiste a una gran transformación del paisaje mediante un profundo proceso deforestador mediado por el fuego y la ganadería extensiva en un periodo de retracción de la agricultura (ESTEBAN, RIERA, 1994). Aunque en principio los elementos de perturbación del bosque parecen ser los mismos, en la costa de Girona no tenemos evidencias de agricultura ni se utiliza el fuego mediante incendios antrópicos como factor de deforestación.

A lo largo de la segunda mitad del siglo XII d.C., y hasta finales del siglo XIV d.C., los bosques se recuperan -tanto a nivel local (litoral) como regional (Pirineo oriental)- del abrupto cambio climático producido con anterioridad, y del fuerte impacto antrópico que tuvo el poblamiento del Pirineo durante el siglo XI d.C. y la primera mitad del siglo XII d.C. sobre las masas forestales pirenaicas, especialmente sobre el pinar. Aunque la presión pastoral se incrementa hacia las zonas costeras, se vuelve a una situación de sostenibilidad semejante a la experimentada entre los siglos VIII y X d.C., donde la ganadería siguen siendo el principal elemento de perturbación de los ecosistemas a expensas de una arboricultura (castaño y olivo) escasamente desarrollada.

De nuevo, a finales del siglo XIII d.C. e inicios del XIV d.C. (I280-I 340 d.C.), una recurrente perturbación climática, un cambio climático abrupto, altera las pautas socioeconómicas del área de estudio ante condiciones relativamente áridas, ralentizándose la presión pastoral y sufriendo cierto detrimento las formaciones forestales. Entre finales del siglo XIV d.C. y la primera mitad del siglo XV d.C. (I380-I 450 d.C.) se produce un nuevo evento climático abrupto que, como antes, se solapa con un fuerte impacto antrópico sobre el paisaje, 
produciéndose cierto detrimento forestal. Sin embargo, este segundo evento, a diferencia del anterior, muestra una presión pastoral mayor, por lo que no siempre la respuesta de las sociedades humanas parece ser la misma a los cambios climáticos, especialmente cuando transcurre poco tiempo entre uno y otro como es este caso. En efecto, en la transición entre los siglos XV-XVI d.C. (I450-I530 d.C.), son los bosques litorales de robles y alcornoques los que sufren un mayor impacto antrópico mediado por la actividad pastoril, mientras que las formaciones forestales pirenaicas no sólo no se ven afectadas sino que comienzan a desarrollarse, aumentando la masa de pinar, reapareciendo el haya y documentándose por primera vez abeto y avellano.

En general, desde un punto de vista social, los siglos XIV-XVI d.C. coinciden con un periodo de crisis demográfica y económica en gran parte de Europa, causada por la incidencia de enfermedades y epidemias, lo cual conduce a una relajación del impacto humano sobre los bosques (DESPRAT et alii, 2003). En Cataluña, la crisis de la Baja Edad Media empieza a vislumbrarse a partir de 1333 d.C. -el primer 'gran año de hambre'-, continuándose entre |347-| 35 | d.C. con las primeras oleadas de la peste negra, extendiéndose hasta finales del siglo XV d.C. con profundas crisis económicas y sociales (RODRÍGUEZ PUERTOLAS, 1982; RIU, 2003; FELIU, 2004). En el registro polínico de Port Lligat estos hechos son claramente perceptibles, pues salvo la incidencia del Mínimo de Spörer, los siglos XIII-XVI d.C. coinciden con un periodo de mantenimiento de los bosques, especialmente en el área pirenaica donde incluso se produce un desarrollo muy importante del bosque de pinos entre los siglos $\mathrm{XV}$ - $\mathrm{XV}$ I d.C., favorecido por el despoblamiento del Pirineo catalán (PĖLACHS, 2004). Tal periodo de crisis profunda provocó una gran movilidad poblacional y el agotamiento de las zonas de cultivo (FELIU, 2004), lo cual paleoambientalmente queda reflejado por un aumento de la antropización del paisaje.

Prácticamente sin interrupción, desde el siglo VIII d.C., el registro fósil de Port Lligat demuestra el cultivo de castaño en la región de estudio, posiblemente también del olivo; mientras que el nogal, la vid, el cáñamo y los cereales sólo empezaron a cultivarse a partir de ca. 1530 d.C. En el caso del cáñamo, los datos aportados por Port Lligat contrastan con el inicio de su cultivo en otras zonas de Cataluña hacia los años 600-650 d.C., aunque es cierto que la máxima difusión de éste parece producirse a partir de ca. 1580 d.C. (RIERA et alii, 2004, 2006); lo cual coincidiría con lo documentado en Port Lligat, que en realidad no estaría más que recogiendo un proceso de cultivo del cáñamo que seguramente tendría una significación a nivel regional que no local en el entorno inmediato del registro. En cuanto a la vid ocurre otro tanto, pues si en Port Lligat sólo aparece documentado su polen a partir de 1530 d.C., en el Llano de Barcelona hay referencia sucinta a su cultivo desde al menos el siglo X d.C. (RIERA, ESTEBAN, I 994; PALET, RIERA, 1997; MAURI, 2006). Es probable, que tanto la vid como el olivo se cultivaran en terrazas en ciertas vertientes de las elevaciones interiores, pues este sistema ha sido igualmente atestiguado en el Llano de Barcelona en diversos periodos medievales entre los siglos XII-XVI d.C. (PALET, RIERA, 1997).

Los datos aportados por Port Lligat coinciden con otros registros polínicos regionales, que demuestran como los siglos XVI-XVII d.C. suponen la mayor explotación forestal de las zonas costeras y pirenaicas, con una presión pastoral amplia y un máximo de la expansión agraria (RIERA, ESTEBAN, 1994; PÈLACHS, 2004; RIERA et alii, 2004, 2006; BURJACHS et alii, 2005; MIRAS et alii, 2007). En Port Lligat, a partir de la segunda mitad del siglo XVI d.C., se cultiva el castaño, el nogal, la vid, el cáñamo y el olivo, y aparecen las primeras evidencias de cerealicultura; actividades éstas que se mantienen hasta al menos la segunda mitad del siglo XIX d.C. en un ámbito local y regional.

Resulta significativo el hecho de que entre 1530 d.C. y 1840 d.C. los bosques pirenaicos de abeto, haya y pino experimenten cierto auge, aun cuando estos territorios estaban siendo sometidos a una carga ganadera intensiva. El 
establecimiento de zonas refugio para dichas especies, particularmente para el pino y el abeto, relacionadas con el desarrollo de carboneras datadas en el Pirineo oriental en estas fechas (PÈLACHS, 2004; MIRAS et alii, 2007), habría permitido su supervivencia y desarrollo sostenible. En el caso del haya, un aumento de la antropización de los ambientes pirenaicos podría haber favorecido la proliferación de esta especie (GALOP, 1998). Sólo a partir del siglo XIX d.C. los bosques de abeto comienzan su declive, cuando la presión pastoral se incrementa de manera considerable.

Al igual que en los siglos anteriores, un nuevo cambio climático abrupto, entre 1645 d.C. y 17I5 d.C., vino a interrumpir la tónica general señalada para los siglos XVII-XVIII d.C. En este pequeño intervalo de tiempo, el advenimiento de condiciones climáticas ciertamente áridas y frías, posiblemente las más drásticas de toda la Pequeña Edad del Hielo, provocó el retraimiento de los bosques de quercíneas caducifolias así como de los abetares y pinares pirenaicos. A su vez, este clima adverso supuso cierto cese del cultivo del castaño y la vid y el fin de toda actividad agrícola versada en los cereales.

A partir de 1850 d.C., con el inicio del periodo industrial, y hasta la actualidad, la presión antrópica sobre los ambientes costeros disminuye, pues dejan de cultivarse el cáñamo y los cereales, lo cual permite la recuperación del alcornocal, el robledal y el encinar/alsinar. En el Pirineo el pinar se recupera igualmente, el hayedo se mantiene aún reduciendo sus porcentajes, mientras que el abeto sufre una regresión muy importante continuando la tónica empezada en la primera mitad del siglo XIX d.C. Particularizando nuestra atención en el haya, hemos de decir que su declive en las últimas décadas, tal y como ha ocurrido en las montañas del Montseny (PEÑUELAS et alii, 2007), parece estar relacionado con el progresivo cambio climático y el aumento de la temperatura, lo cual está provocando el detrimento del hayedo a favor de formaciones perennifolias de Quercus ilex. En el registro polínico de Port Lligat estos hechos son muy evidentes a partir de ca.
1950 d.C., aunque la quercínea que se muestra preponderante es el roble que no la alsina.

En resumen, dinámica antrópica y cambios climáticos son elementos de diagnosis paleoecológica que se solapan, no siendo siempre factible determinar la importancia de uno $u$ otro en el registro fósil. En este trabajo, no obstante, hemos intentado aportar algunas connotaciones de tipo paleoclimático para ayudar a entender no sólo el registro paleoambiental sino también su correspondencia con el histórico. Reconocemos las limitaciones de esta aproximación, ya señaladas antes, pero hemos creído conveniente hacerlo así para lograr un lectura histórica del paleoambiente más entendible.

\section{BIBLIOGRAFÍA}

ANDERSON, R.S.; HOMOLA, L.; DAVIS, R.B.; JACOBSON, G.L. (1984): "Fossil remains of the mycorrhizal fungal Glomus fasciculatum complex in postglacial lake sediments from Maine", Canadian Journal of Botany, 62, pp. 2325-2328.

BEAULIEU DE, J.L. ; MIRAS,Y.; ANDRIEU-PONEL,V.; GUITER, F. (2005): "Vegetation dynamics in north-western Mediterranean regions: Instability of the Mediterranean bioclimate", Plant Biosystems, I39, pp. I|4- 26.

BEHRE, K.E. (| 198I): "The interpretation of anthropogenic indicators in pollen diagrams", Pollen et Spores, 23, pp. 225-245.

BONNASSIE, P. (1988): Cataluña mil años atrás (siglos X-XI). Península Ediciones 62, Barcelona.

BONNASSIE, P. (1990): La Catalogne au tournant de l'an mil: croissance et mutations d'une société. Albin Michel, París.

BURJACHS, F. (2004): "Paisatges i climes medievals de la façan mediterrània ibèrica nord-occidental", Actes del Congrès Castells medievals a la Mediterrània nord-occidental. Museu Etnològic del Montseny, Arbúcies, pp. 23I-246.

BURJACHS, F.; BACH, J.; BUXÓ, R. ; LLACER, P.; MACGLADE, J. ; PICAZO, M. ; PIQUÉ, R. ; ROS, M.T. (2005) : "El territori d'Emporion i les seves dades paleoambientals", Empúries, 54, pp. 25-32.

BURJACHS, F. ; ESTEBAN, A. ; GÉNOVA, R. ; JULIÀ, R. ; LLASAT, C.; MONTSERRAT, J.; PARRA, I. ; PÉREZ OBIOL, R. ; ROURE, J.M. ; VILAPLANA, J.M. (1990) : "Vegetación y paleoclimatología en el N.E. peninsular durante el Tardi y Postglacial", Polen, esporas y sus aplicaciones. Universidad de Granada, Granada, pp. 465-470. 
BURJACHS, F.; SCHULTE, L. (2003): "El paisatge vegetal del Penedès entre la Prehistòria i el Món Antic", Territoris antics a la M editerrània i a la Cossetània oriental. Generalitat de Catalunya, Barcelona, pp. 249-254.

CARRIÓN, J.S., NAVARRO, C. (2002): "Cryptogam spores and other non-pollen microfossils as sources of palaeoecological information: case-studies from Spain", Annales Botanici Fennici, 39, pp. I-14.

CONEDERA, M.; KREBS, P.; TINNER, W.; PRADELLA, M.; TORRIANI, D. (2004): "The cultivation of Castanea sativa (Mill.) in Europe, from its origin to its diffusion on a continental scale", Vegetation H istory and Archaeobotany, 13, pp. $161-179$.

DESPRAT, S. ; SÁNCHEZ-GOÑI, M.F. ; LOUTRE, M.F. (2003) : "Revealing climatic variability of the last three millennia in northwestern Iberia using pollen influx data", Earth and Planetary Science Letters, 213, pp. 63-78.

ESTEBAN, A.; RIERA, S. (1994): "Transformacions del paisatge i ramaderia a la costa catalana del Penedès i Garraf (Barcelona) a l'alta edat mitja", IV Congreso de Arqueología Medieval Española. Sociedades en Transición, octubre de 1993. Alacant, pp. 647-655.

FAEGRI, K.; IVERSEN, J. (1989): Textbook of Pollen Analysis. John Wiley \& Sons, Chichester.

FELIU, G. (2004): "La crisis catalana de la Baja Edad Media : estado de la cuestión”, H ispania, 64, pp. 435-466.

FRANQUESA, t. (1995): El paisatge vegetal de la península del Cap de Creus. Institut d'Estudis Catalans, Barcelona.

GALOP, D. (1998): La forêt, l'homme et le troupeau dans les Pyrénées. 6000 ans d'histoire de l'environnement entre Garonne et M éditerranée. GEODE, Laboratoire d'Ecologie Terrestre et FRAMESPA, Toulouse.

GRIMM, E. (1992) : Tilia, version 2. USA Illinois State Museum, Research and Collection Center, Springfield.

IZQUIERDO BENITO, R. ( 1994) : "La Arqueología medieval en España: antecedentes y estado actual", Arqueología y Territorio M edieval, I, pp. I19-127.

LÓPEZ SÁEZ, J.A.; LÓPEZ GARCÍA, P.; BURJACHS, F. (2003): "Arqueopalinología: Síntesis Crítica", Polen, I2, pp. 5-35.

LÓPEZ SÁEZ, J.A.; VAN GEEL, B.; FARBOS-TEXIER, S.; DIOT, M.F. (1998): "Remarques paléoécologiques à propos de quelques palynomorphes non-polliniques provenant de sédiments quaternaires en France", Revue de Paléobiologie, 17, pp. 445-459.

LÓPEZ SÁEZ, J.A.; VAN GEEL, B.; MARTÍN SÁNCHEZ, M. (2000): "Aplicación de los microfósiles no polínicos en Palinología Arqueológica", Contributos das Ciências e das Technologias para a Arqueologia da Península Ibérica. Actas 30 Congresso de Arqueologia Peninsular. Adecap, Porto, pp. II-20.

MALLARACH, J.M.; PÉREZ OBIOL, R.; ROURE, J.M. ( I985): "Aportaciones al conocimiento del clima y la vegetación durante el Cuaternario reciente, en el N.E. de la Península Ibérica", Actas I Reunión del Cuaternario Ibérico. G.E.T.C.G.T.P.E.Q, Lisboa, pp. 201-212.
MATEO, M.A.: RENOM, P.; JULİ̀, R.: ROMERO, l:; MICHENER, R. (2002): "An unexplored sedimentary record for the study of environmental change in Mediterranean environments: Posidonia oceanica (L.) Delile peats", The study of environmental change using isotope techniques. International Atomic Energy Agency C\&S Papers Series I6P, Vienna, pp. I63-173.

MATEO, M.A.; ROMERO, J.; PÉREZ, M.; LITTLER, M.M.; LITTLER, D.S. (1997): "Dynamics of millenary organic deposits resulting from the growth of the mediterranean seagrass Posidonia oceanica", Estuarine, Coastal and Shelf Science, 44, pp. 103-1 10.

MAURI, A. (2006): La configuració del paisatge medieval: el comtat de Barcelona find el segle XI. Universitat de Barcelona, Barcelona.

MENCHON I BES, J. (1998): "Necrópolis altomedievales y despoblación en la provincia de Tarragona, el caso de la Conca de Barberà", Arqueología y Territorio Medieval, 5 , pp. 5-29.

MIRAS,Y.; EJARQUE, A.; RIERA, S.; PALET, J.M.; ORENGO, H.; EUBA, I. (2007): "Dynamique holocène de la végétation et occupation des Pyrénées andorranes depuis le Néolithique ancien, d'après l'analyse pollinique de la tourbière de Bosc dels Estanyons (2180 m, Vall del Madriu, Andorre)", Comptes Rendus Palevol, 6, pp. 291-300.

PALET, J.M. ; RIERA, S. (1994): "Landscape dynamics from Iberian-Roman (2nd.- I st. centuries BC) to Early Medieval times (I 2th. Century) in the Montjuïc-El Port sector (Plain of Barcelona, NE Iberian Peninsula)", Archeologia Medievale, 21 , pp. 517-540.

PALET, J.M. ; RIERA, S. ( 1997) : "Changements du paysage dans la plaine de Barcelone (Catalogne) de la Protohistoire au Moyen Âge", La dynamique des paysages protohistoriques, antiques, médiévaux et modernes. XVIle Rencontres Internationales d'Archéologie et d'H istoire d'Antibes. Éditions APDCA, Sophia-Antipolis, pp. 259-270.

PANTALEÓN-CANO, J.; PÉREZ-OBIOL, R.; ROURE, J.M. (1994):"La representación del paisjae vegetal del área de Barcelona durante el último milenio a partir del análisis polínico de sedimentos marinos del delta del río Besós datados por $210 \mathrm{~Pb}$ ", Trabajos de Palinología Básica y Aplicada. Universitat de València, València, pp. 2 I I-223.

PELACHS, A. (2004): Deu mil anys de geohistòria ambiental al Pirineu central català. Universitat Autònoma de BarceIona, Bellaterra.

PEÑUELAS, J.; OGAYA, R.; BOADA, M.; JUMP, A. (2007): "Migration, invasion and decline: changes in recruitment and forest structure in a warming-linked shift of European beech forest in Catalonia (NE Spain)", Ecography, 30, pp. 829-837.

PÉREZ OBIOL, R.; ROURE, J.M.; VILAR, L. (|99|): "History of some plant communities of the Sils zone ( $\mathrm{La}$ Selva, Catalonia, Spain) based on pollen analysis", Scientia gerundensis, 17, pp. 37-44.

RIERA, S. (1999): "Evolució del paisatge al Pla de Barcelona", Del Romà al Romànic. Enciclopèdia Catalana, Barcelona, pp. I36- 137. 
RIERA, S., ESTEBAN, A. (1994): "Vegetation history and human activity during the last 6000 years on the central Catalan coast (northeastern Iberian Peninsula)", Vegetation $\mathrm{H}$ istory and Archaeobotany, 3, pp. 7-23.

RIERA, S.; LÓPEZ SÁEZ, J.A.; JULIÀ, R. (2006): "Lake responses to historical land use changes in northern Spain:The contribution of non-pollen palynomorphs in a multiproxy study", Review of Palaeobotany and Palynology, I4I, pp. 127-137.

RIERA, S. ; PALET, J.M. (1993) : "Evolució del sector de Montjüic-el Port entre l'època romana i altmedieval (s. III-X): una contribució a l'estudi diacrònic del paisatge", Actes del III Congrès d'Història del Pla de Barcelona. Barcelona, pp. 49-70.

RIERA, S.; WANSARD, G.; JULIÀ, R. (2004): "2000-year environmental history of a karstic lake in the Mediterranean Pre-Pyrenees: the Estanya lakes (Spain)", Catena 55, pp. 293-324.
RIU, M., 2003. "La vivienda rural en el Prepirineo y Pirineo catalán (siglos XI al XVI)", Scripta Nova, 7 ( I46), pp. I-5.

RIVAS MARTÍNEZ, S. (1987): M emoria del MAPA de Series de Vegetación de España. Ministerio de Agricultura, Pesca y Alimentación, ICONA, Madrid.

RODRÍGUEZ PUERTOLAS, J. (1982): "La crisis de la Baja Edad Media calatana y la poesía de la época", Actas del Cuarto Congreso Internacional de Hispanistas. Universidad de Salamanca, Salamanca, pp. 487-498.

ROMERO, J.; PÉREZ, M.; MATEO, M.A.; SALA, E. (1994): "The below ground organs of the Mediterranean seagrass Posidonia oceanica as a biogeochemical sink", Aquatic Botany, 47, pp. 13-19.

VAN GEEL, B. (200 I): "Non-pollen palynomorphs", Tracking environmental change using lake sediment, Volume 3 : Terrestrial, Algal, and Siliceous Indicators. Kluwer Academic Publishers, Dordrecht, pp. 99-119. 


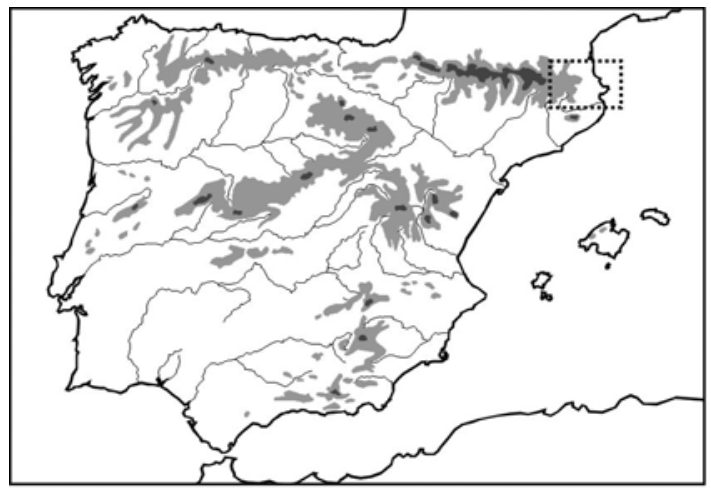

Fig. 1. Mapa de situación de la Bahía de Port Lligat en el NE de la Península Ibérica y localización del sondeo palinológico en la mata de Posidonia oceanica.
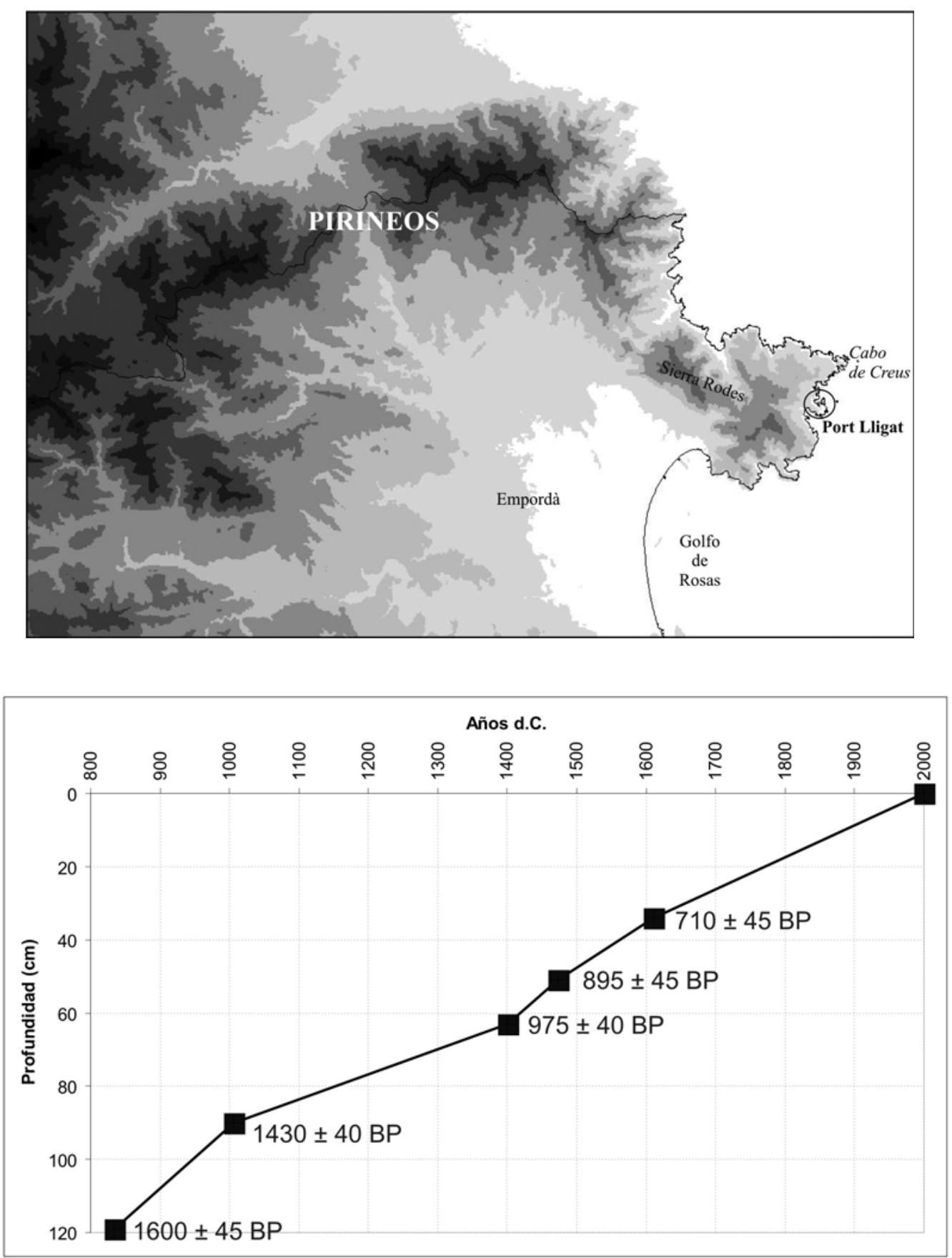

Fig. 2. Modelo cronológico del registro sedimentario de Port Lligat. 


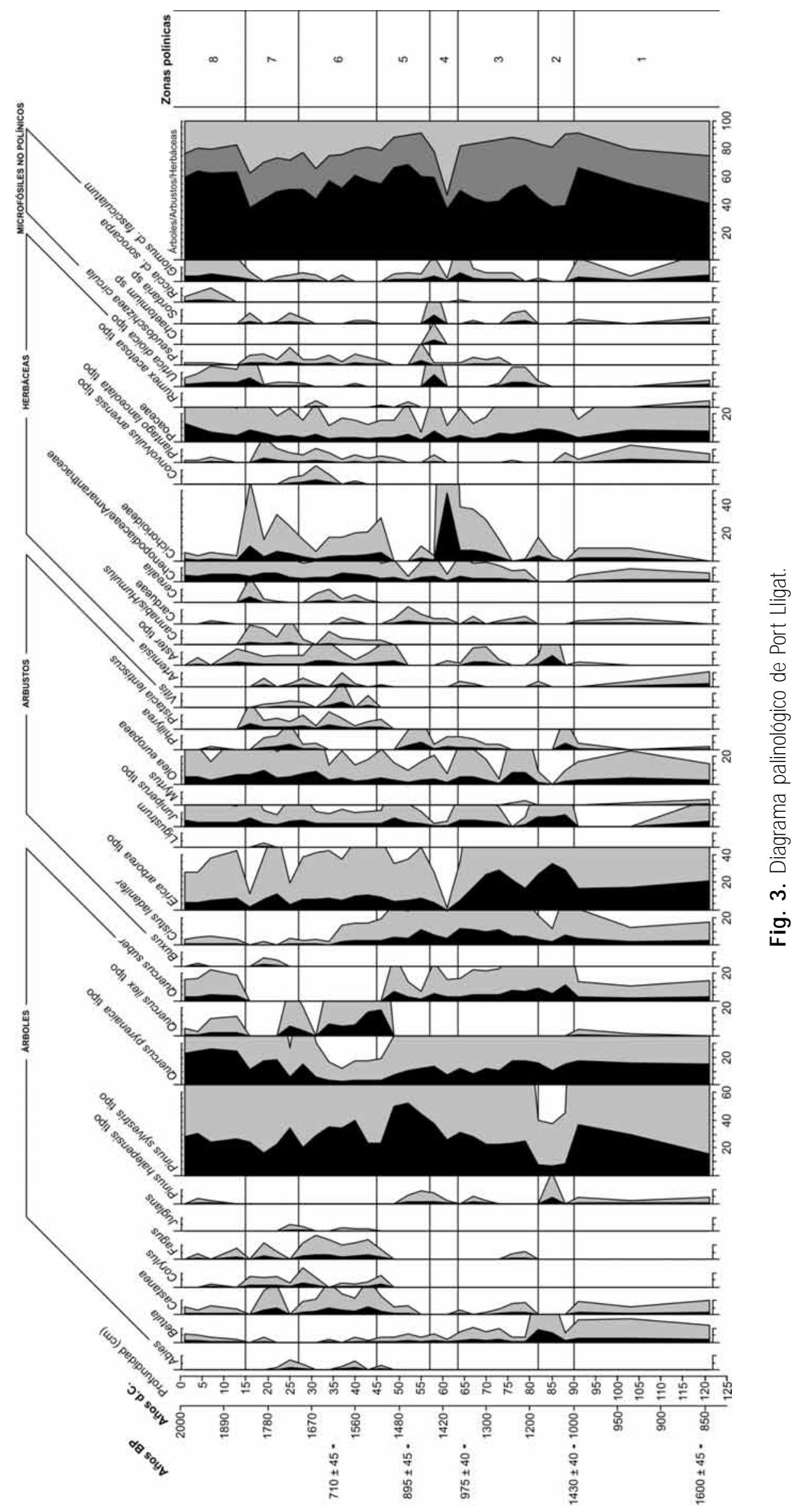

